TRANSACTIONS OF THE

AMERICAN MATHEMATICAL SOCIETY

Volume 361, Number 4, April 2009, Pages 1885-1902

S 0002-9947(08)04510-8

Article electronically published on November 25, 2008

\title{
HOMOTOPY ON SPATIAL GRAPHS AND THE SATO-LEVINE INVARIANT
}

\author{
THOMAS FLEMING AND RYO NIKKUNI
}

\begin{abstract}
Edge-homotopy and vertex-homotopy are equivalence relations on spatial graphs which are generalizations of Milnor's link-homotopy. We introduce some edge (resp. vertex)-homotopy invariants of spatial graphs by applying the Sato-Levine invariant for the 2-component constituent algebraically split links and show examples of non-splittable spatial graphs up to edge (resp. vertex)-homotopy, all of whose constituent links are link-homotopically trivial.
\end{abstract}

\section{INTRODUCTION}

Throughout this paper we work in the piecewise linear category. Let $G$ be a finite graph which does not have isolated vertices and free vertices. An embedding $f$ of $G$ into the 3 -sphere $S^{3}$ is called a spatial embedding of $G$ or simply a spatial graph. For a spatial embedding $f$ and a subgraph $H$ of $G$ which is homeomorphic to the 1-sphere $S^{1}$ or a disjoint union of 1-spheres, we call $f(H)$ a constituent knot or a constituent link of $f$, respectively. A graph $G$ is said to be planar if there exists an embedding of $G$ into the 2-sphere $S^{2}$, and a spatial embedding of a planar graph is said to be trivial if it is ambient isotopic to an embedding of the graph into a 2-sphere in $S^{3}$. A spatial embedding $f$ of a graph $G$ is said to be split if there exists a 2-sphere $S$ in $S^{3}$ such that $S \cap f(G)=\emptyset$ and each component of $S^{3}-S$ has intersection with $f(G)$, and otherwise $f$ is said to be non-splittable.

Two spatial embeddings of a graph $G$ are said to be edge-homotopic if they are transformed into each other by self crossing changes and ambient isotopies, where a self crossing change is a crossing change on the same spatial edge, and vertex-homotopic if they are transformed into each other by crossing changes on two adjacent spatial edges and ambient isotopies 1 These equivalence relations were introduced by Taniyama [19] as generalizations of Milnor's link-homotopy on links 8; namely if $G$ is homeomorphic to a disjoint union of 1-spheres, then these are none other than link-homotopy. There are many studies about link-homotopy. In particular, the link-homotopy classification was given for 2- and 3-component links

Received by the editors August 31, 2005 and, in revised form, March 10, 2007.

2000 Mathematics Subject Classification. Primary 57M15; Secondary 57M25.

Key words and phrases. Spatial graph, edge-homotopy, vertex-homotopy, Sato-Levine invariant.

The first author was supported by a Fellowship of the Japan Society for the Promotion of Science for Post-Doctoral Foreign Researchers (Short-Term) (No. PE05003).

The second author was partially supported by a Grant-in-Aid for Scientific Research (B) (2) (No. 15340019), Japan Society for the Promotion of Science.

${ }^{1}$ In [19], edge-homotopy and vertex-homotopy were called homotopy and weak homotopy, respectively.

(C)2008 American Mathematical Society 
by Milnor [8, for 4-component links by Levine [7] and for all links by Habegger and Lin [2]. On the other hand, there are very few studies about edge (resp. vertex)homotopy on spatial graphs [18, 9], 13], [1].

In [18, Taniyama defined an edge (resp. vertex)-homotopy invariant of spatial graphs called the $\alpha$-invariant by applying the Casson invariant (or equivalently the second coefficient of the Conway polynomial) of the constituent knots and showed that there exists a non-trivial spatial embedding $f$ of a planar graph up to edge (resp. vertex)-homotopy, even in the case where $f$ does not contain any constituent link. But the $\alpha$-invariant cannot detect a non-splittable spatial embedding of a disconnected graph up to edge (resp. vertex)-homotopy. As far as the authors know, an example of a non-splittable spatial embedding of a disconnected graph up to edge (resp. vertex)-homotopy, all of whose constituent links are link-homotopically trivial, has not yet been demonstrated.

Our purpose in this paper is to study spatial embeddings of disconnected graphs up to edge (resp. vertex)-homotopy by applying the Sato-Levine invariant [14] (or equivalently the third coefficient of the Conway polynomial) for the constituent 2component algebraically split links and show that there exist infinitely many nonsplittable spatial embeddings of a certain disconnected graph up to edge (resp. vertex)-homotopy, all of whose constituent links are link-homotopically trivial. These examples show that edge (resp. vertex)-homotopy on spatial graphs behaves quite differently than link-homotopy on links. In the next section we give the definitions of our invariants and state their invariance up to edge (resp. vertex)homotopy.

\section{Definitions of InVARIANts}

We call a subgraph of a graph $G$ a cycle if it is homeomorphic to the 1-sphere, and a cycle is called a $k$-cycle if it contains exactly $k$ edges. For a subgraph $H$ of $G$, we denote the set of all cycles of $H$ by $\Gamma(H)$. We set $\mathbb{Z}_{m}=\{0,1, \ldots, m-1\}$ for a positive integer $m$ and $\mathbb{Z}_{0}=\mathbb{Z}$. We regard $\mathbb{Z}_{m}$ as an abelian group in the obvious way. We call a map $\omega: \Gamma(H) \rightarrow \mathbb{Z}_{m}$ a weight on $\Gamma(H)$ over $\mathbb{Z}_{m}$. For an edge $e$ of $H$, we denote the set of all cycles of $H$ which contain the edge $e$ by $\Gamma_{e}(H)$. For a pair of two adjacent edges $e_{1}$ and $e_{2}$ of $H$, we denote the set of all cycles of $H$ which contain the edges $e_{1}$ and $e_{2}$ by $\Gamma_{e_{1}, e_{2}}(H)$. Then we say that a weight $\omega$ on $\Gamma(H)$ over $\mathbb{Z}_{m}$ is weakly balanced 2 on an edge $e$ if

$$
\sum_{\gamma \in \Gamma_{e}(H)} \omega(\gamma)=0
$$

in $\mathbb{Z}_{m}$ [10], and weakly balanced on a pair of adjacent edges $e_{1}$ and $e_{2}$ if

$$
\sum_{\gamma \in \Gamma_{e_{1}, e_{2}}(H)} \omega(\gamma)=0
$$

in $\mathbb{Z}_{m}$. Let $G=G_{1} \cup G_{2}$ be a disjoint union of two connected graphs and $\omega_{i}$ : $\Gamma\left(G_{i}\right) \rightarrow \mathbb{Z}_{m}$ a weight on $\Gamma\left(G_{i}\right)$ over $\mathbb{Z}_{m}(i=1,2)$. Let $f$ be a spatial embedding of $G$ such that

$$
\omega_{1}(\gamma) \omega_{2}\left(\gamma^{\prime}\right) \operatorname{lk}\left(f(\gamma), f\left(\gamma^{\prime}\right)\right)=0
$$

\footnotetext{
${ }^{2}$ A weight $\omega$ on $\Gamma(H)$ over $\mathbb{Z}_{m}$ is said to be balanced on an edge e of $H$ if $\sum_{\gamma \in \Gamma_{e}(H)} \omega(\gamma)[\gamma]=0$ in $H_{1}\left(H ; \mathbb{Z}_{m}\right)$, where the orientation of $\gamma$ is induced by the one of $e$ [18.
} 
in $\mathbb{Z}$ for any $\gamma \in \Gamma\left(G_{1}\right)$ and $\gamma^{\prime} \in \Gamma\left(G_{2}\right)$, where $\operatorname{lk}(L)=\operatorname{lk}\left(K_{1}, K_{2}\right)$ denotes the linking number of a 2-component oriented link $L=K_{1} \cup K_{2}$. Then we define $\beta_{\omega_{1}, \omega_{2}}(f) \in \mathbb{Z}_{m}$ by

$$
\beta_{\omega_{1}, \omega_{2}}(f) \equiv \sum_{\substack{\gamma \in \Gamma\left(G_{1}\right) \\ \gamma^{\prime} \in \Gamma\left(G_{2}\right)}} \omega_{1}(\gamma) \omega_{2}\left(\gamma^{\prime}\right) a_{3}\left(f(\gamma), f\left(\gamma^{\prime}\right)\right) \quad(\bmod m),
$$

where $a_{3}(L)=a_{3}\left(K_{1}, K_{2}\right)$ denotes the third coefficient of the Conway polynomial of a 2-component oriented link $L=K_{1} \cup K_{2}$. We remark here that $a_{3}(L)$ coincides with the Sato-Levine invariant $\beta(L)$ of $L$ if $L$ is algebraically split, namely $\operatorname{lk}\left(K_{1}, K_{2}\right)=0$ [1, [17. Thus our $\beta_{\omega_{1}, \omega_{2}}(f)$ is also the modulo $m$ reduction of the summation of Sato-Levine invariants for the constituent 2 -component algebraically split links of $f$.

Remark 2.1. For a 2-component algebraically split link $L=K_{1} \cup K_{2}$,

(1) The value of $a_{3}(L)$ does not depend on the orientations of $K_{1}$ and $K_{2}$. Actually we can check it easily by the original definition of the Sato-Levine invariant.

(2) The value of $a_{3}(L)$ is not a link-homotopy invariant of $L$ (see also Lemma 3.1). For example, the Whitehead link $L$ is link-homotopically trivial but $a_{3}(L)=1$.

Now we state the invariance of $\beta_{\omega_{1}, \omega_{2}}$ up to edge (resp. vertex)-homotopy under some conditions on the graphs.

Theorem 2.2. Let $G=G_{1} \cup G_{2}$ be a disjoint union of two connected graphs and $\omega_{i}$ a weight on $\Gamma\left(G_{i}\right)$ over $\mathbb{Z}_{m}(i=1,2)$. Let $f$ be a spatial embedding of $G$ such that

$$
\omega_{1}(\gamma) \omega_{2}\left(\gamma^{\prime}\right) \operatorname{lk}\left(f(\gamma), f\left(\gamma^{\prime}\right)\right)=0
$$

in $\mathbb{Z}$ for any $\gamma \in \Gamma\left(G_{1}\right)$ and $\gamma^{\prime} \in \Gamma\left(G_{2}\right)$. Then we have the following:

(1) If $\omega_{i}$ is weakly balanced on any edge of $G_{i}(i=1,2)$, then $\beta_{\omega_{1}, \omega_{2}}(f)$ is an edge-homotopy invariant of $f$.

(2) If $\omega_{i}$ is weakly balanced on any pair of adjacent edges of $G_{i}(i=1,2)$, then $\beta_{\omega_{1}, \omega_{2}}(f)$ is a vertex-homotopy invariant of $f$.

We prove Theorem 2.2 in the next section. In addition, by using an integer-valued invariant (Theorem 4.2), we show that there exist infinitely many non-splittable spatial embeddings of a certain disconnected graph up to edge-homotopy all of whose constituent links are link-homotopically trivial (Example 4.3). We also exhibit an infinite family of non-splittable spatial embeddings of a certain disconnected graph up to vertex-homotopy which can be distinguished by our integer-valued invariant (Example 4.4).

We note that if a graph $G$ contains a connected component which is homeomorphic to the 1-sphere, then our invariants in Theorem 2.2 are useless. For such cases, we can define edge (vertex)-homotopy invariants that take values in $\mathbb{Z}_{2}$ on a weaker condition for weights than the one stated in Theorem 2.2. For a subgraph $H$ of a graph $G$, we say that a weight $\omega$ on $\Gamma(H)$ over $\mathbb{Z}_{2}$ is totally balanced if

$$
\sum_{\gamma \in \Gamma(H)} \omega(\gamma)[\gamma]=0
$$


in $H_{1}\left(H ; \mathbb{Z}_{2}\right)$. We note that if a weight $\omega$ on $\Gamma(H)$ over $\mathbb{Z}_{2}$ is totally balanced, then it is weakly balanced on any edge $e$ of $H$ (Lemma 3.2), but not always weakly balanced on any pair of adjacent edges of $H$ (Remark 3.3). Then we have the following:

Theorem 2.3. Let $G=G_{1} \cup G_{2}$ be a disjoint union of two connected graphs and $\omega_{i}$ a weight on $\Gamma\left(G_{i}\right)$ over $\mathbb{Z}_{2}(i=1,2)$. Let $f$ be a spatial embedding of $G$ such that

$$
\omega_{1}(\gamma) \omega_{2}\left(\gamma^{\prime}\right) \operatorname{lk}\left(f(\gamma), f\left(\gamma^{\prime}\right)\right)=0
$$

in $\mathbb{Z}$ for any $\gamma \in \Gamma\left(G_{1}\right)$ and $\gamma^{\prime} \in \Gamma\left(G_{2}\right)$. Then we have the following:

(1) If either $\omega_{1}$ is totally balanced on $\Gamma\left(G_{1}\right)$ or $\omega_{2}$ is totally balanced on $\Gamma\left(G_{2}\right)$, then $\beta_{\omega_{1}, \omega_{2}}(f)$ is an edge-homotopy invariant of $f$.

(2) If either $\omega_{1}$ is totally balanced on $\Gamma\left(G_{1}\right)$ and weakly balanced on any pair of adjacent edges of $G_{1}$, or $\omega_{2}$ is totally balanced on $\Gamma\left(G_{2}\right)$ and weakly balanced on any pair of adjacent edges of $G_{2}$, then $\beta_{\omega_{1}, \omega_{2}}(f)$ is a vertex-homotopy invariant of $f$.

We also prove Theorem 2.3 in the next section and give some examples in Section 5. In particular, we show that there exist infinitely many non-splittable spatial embeddings of a certain disconnected graph up to vertex-homotopy, all of whose constituent links are link-homotopically trivial (Example 5.4). We remark here that the $\mathbb{Z}_{2}$-valued invariant in Theorem 2.3 cannot always be extended to an integervalued one (Remark 5.5).

Theorems 2.2 and 2.3 do not work for spatial graphs as illustrated in Figure 2.1. for instance. In Section 6, we state a method to detect such non-splittable spatial graphs up to edge-homotopy by using a planar surface having a graph as a spine (Theorem 6.1). Actually we show that each of the spatial graphs as illustrated in Figure 2.1 is non-splittable up to edge-homotopy (Example 6.2).

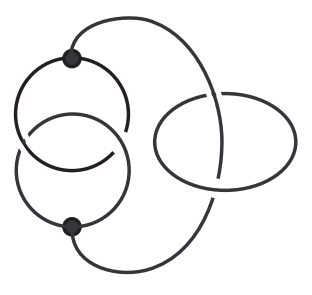

(1)

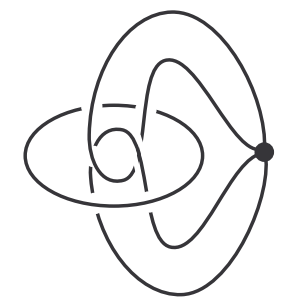

(2)

Figure 2.1

\section{Proofs of Theorems 2.2 and 2.3}

We first calculate the change in the third coefficient of the Conway polynomial of 2-component algebraically split links which differ by a single self crossing change. 
Lemma 3.1. Let $L_{+}$and $L_{-}$be two 2-component oriented links and $L_{0}=J_{1} \cup$ $J_{2} \cup K$ a 3-component oriented link which are identical except inside the depicted regions as illustrated in Figure 3.1. Suppose that $\operatorname{lk}\left(L_{+}\right)=\operatorname{lk}\left(L_{-}\right)=0$. Then it holds that

$$
a_{3}\left(L_{+}\right)-a_{3}\left(L_{-}\right)=-\mathrm{lk}\left(J_{1}, K\right)^{2}=-\mathrm{lk}\left(J_{2}, K\right)^{2} .
$$
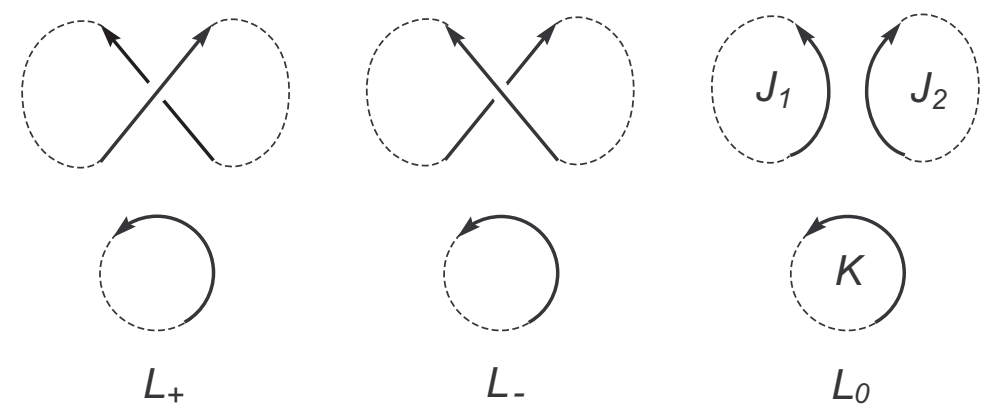

FiguRe 3.1

Proof. By the skein relation of the Conway polynomial and a well-known formula for the second coefficient of the Conway polynomial of a 3-component oriented link (cf. 4], 3], [5]), we have that

$$
\begin{aligned}
a_{3}\left(L_{+}\right)-a_{3}\left(L_{-}\right)= & \operatorname{lk}\left(J_{1}, J_{2}\right) \operatorname{lk}\left(J_{2}, K\right)+\operatorname{lk}\left(J_{2}, K\right) \operatorname{lk}\left(J_{1}, K\right) \\
& +\operatorname{lk}\left(J_{1}, K\right) \operatorname{lk}\left(J_{1}, J_{2}\right) .
\end{aligned}
$$

We note that

$$
\operatorname{lk}\left(J_{1}, K\right)+\operatorname{lk}\left(J_{2}, K\right)=0
$$

by the condition $\operatorname{lk}\left(L_{+}\right)=\operatorname{lk}\left(L_{-}\right)=0$. Thus by (3.1) and (3.2), we have that

$$
\begin{aligned}
a_{3}\left(L_{+}\right)-a_{3}\left(L_{-}\right)= & \operatorname{lk}\left(J_{1}, J_{2}\right)\left\{-\operatorname{lk}\left(J_{1}, K\right)\right\}+\operatorname{lk}\left(J_{2}, K\right) \operatorname{lk}\left(J_{1}, K\right) \\
& +\operatorname{lk}\left(J_{1}, K\right) \operatorname{lk}\left(J_{1}, J_{2}\right) \\
= & \operatorname{lk}\left(J_{2}, K\right) \operatorname{lk}\left(J_{1}, K\right) .
\end{aligned}
$$

Therefore by (3.2) we have the result.

Proof of Theorem [2.2. (1) Let $f$ and $g$ be two spatial embeddings of $G$ such that

$$
\omega_{1}(\gamma) \omega_{2}\left(\gamma^{\prime}\right) \operatorname{lk}\left(f(\gamma), f\left(\gamma^{\prime}\right)\right)=0
$$

in $\mathbb{Z}$ for any $\gamma \in \Gamma\left(G_{1}\right)$ and $\gamma^{\prime} \in \Gamma\left(G_{2}\right)$ and $g$ is edge-homotopic to $f$. Then it also holds that

$$
\omega_{1}(\gamma) \omega_{2}\left(\gamma^{\prime}\right) \operatorname{lk}\left(g(\gamma), g\left(\gamma^{\prime}\right)\right)=0
$$

in $\mathbb{Z}$ for any $\gamma \in \Gamma\left(G_{1}\right)$ and $\gamma^{\prime} \in \Gamma\left(G_{2}\right)$ because the linking number of a 2-component constituent link of a spatial graph is an edge-homotopy invariant. First we show that if $f$ is transformed into $g$ by self crossing changes on $f\left(G_{1}\right)$ and ambient isotopies, then $\beta_{\omega_{1}, \omega_{2}}(f)=\beta_{\omega_{1}, \omega_{2}}(g)$. It is clear that any link invariant of a constituent link of a spatial graph is also an ambient isotopy invariant of the spatial graph. Thus we may assume that $g$ is obtained from $f$ by a single crossing change on $f(e)$ for 
an edge $e$ of $G_{1}$ as illustrated in Figure 3.2. Moreover, by smoothing this crossing point we can obtain the spatial embedding $h$ of $G$ and the knot $J_{h}$ as illustrated in Figure 3.2. Then by (3.3), (3.4), Lemma 3.1 and the assumption for $\omega_{1}$ we have that

$$
\begin{aligned}
\beta_{\omega_{1}, \omega_{2}}(f)-\beta_{\omega_{1}, \omega_{2}}(g) & \equiv \sum_{\substack{\gamma \in \Gamma\left(G_{1}\right) \\
\gamma^{\prime} \in \Gamma\left(G_{2}\right)}} \omega_{1}(\gamma) \omega_{2}\left(\gamma^{\prime}\right)\left\{a_{3}\left(f(\gamma), f\left(\gamma^{\prime}\right)\right)-a_{3}\left(g(\gamma), g\left(\gamma^{\prime}\right)\right)\right\} \\
& =\sum_{\substack{\gamma \in \Gamma_{e}\left(G_{1}\right) \\
\gamma^{\prime} \in \Gamma\left(G_{2}\right)}} \omega_{1}(\gamma) \omega_{2}\left(\gamma^{\prime}\right)\left\{a_{3}\left(f(\gamma), f\left(\gamma^{\prime}\right)\right)-a_{3}\left(g(\gamma), g\left(\gamma^{\prime}\right)\right)\right\} \\
& =-\sum_{\substack{\gamma \in \Gamma_{e}\left(G_{1}\right) \\
\gamma^{\prime} \in \Gamma\left(G_{2}\right)}} \omega_{1}(\gamma) \omega_{2}\left(\gamma^{\prime}\right) \operatorname{lk}\left(h\left(\gamma^{\prime}\right), J_{h}\right)^{2} \\
& =-\left(\sum_{\gamma \in \Gamma_{e}\left(G_{1}\right)} \omega_{1}(\gamma)\right) \sum_{\gamma^{\prime} \in \Gamma\left(G_{2}\right)} \omega_{2}\left(\gamma^{\prime}\right) \operatorname{lk}\left(h\left(\gamma^{\prime}\right), J_{h}\right)^{2} \\
& \equiv 0 .
\end{aligned}
$$

Therefore we have that $\beta_{\omega_{1}, \omega_{2}}(f)=\beta_{\omega_{1}, \omega_{2}}(g)$. In the same way we can show that if $f$ is transformed into $g$ by self crossing changes on $f\left(G_{2}\right)$ and ambient isotopies, then $\beta_{\omega_{1}, \omega_{2}}(f)=\beta_{\omega_{1}, \omega_{2}}(g)$. Thus we have that $\beta_{\omega_{1}, \omega_{2}}$ is an edge-homotopy invariant.

(2) By considering the triple of spatial embeddings as illustrated in Figure 3.3. we can prove (2) in a similar way as the proof of (1). We omit the details.
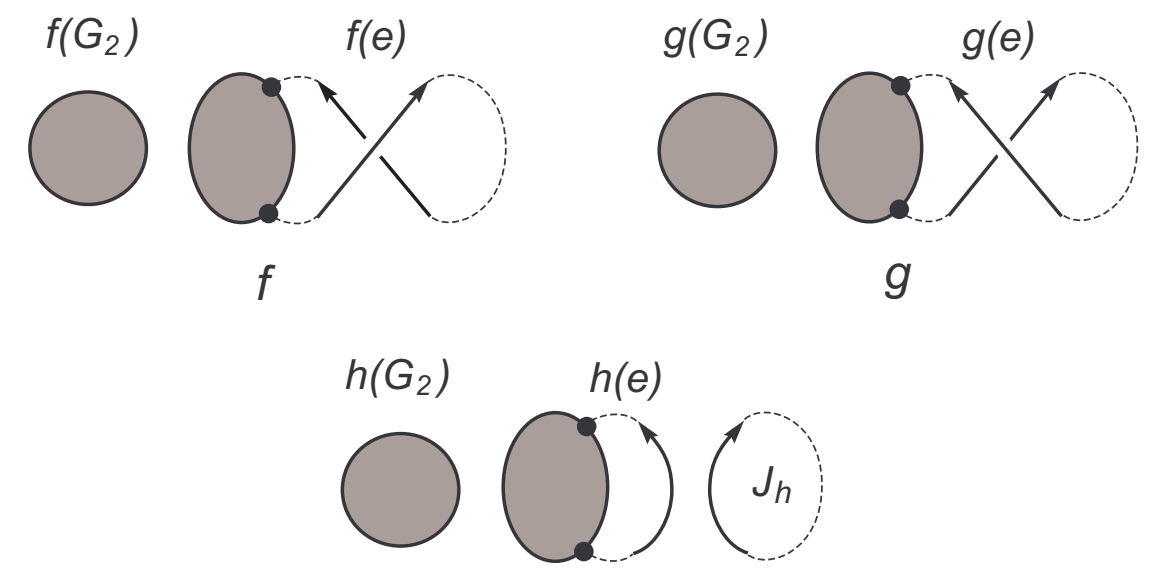

$h$

FiguRE 3.2

Next we prove Theorem 2.3 For a subgraph $H$ of a graph $G$, we have the following.

Lemma 3.2. A totally balanced weight $\omega$ on $\Gamma(H)$ over $\mathbb{Z}_{2}$ is weakly balanced on any edge $e$ of $H$. 

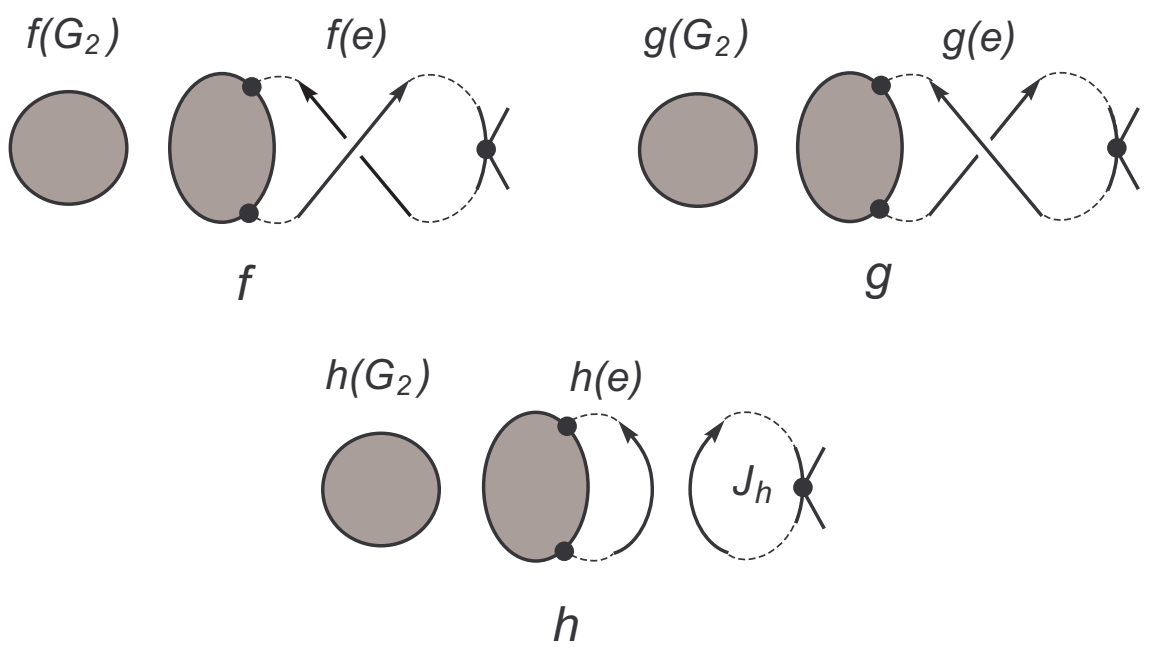

FiguRE 3.3

Proof. For an edge $e$ of $H$, we can represent any $\gamma \in \Gamma_{e}(H)$ as $e+c_{\gamma} \in Z_{1}\left(H ; \mathbb{Z}_{2}\right)$, where $c_{\gamma}$ is a 1 -chain in $C_{1}\left(H \backslash e ; \mathbb{Z}_{2}\right)$. Then we have that

$$
\begin{aligned}
0 & =\sum_{\gamma \in \Gamma(H)} \omega(\gamma)[\gamma] \\
& =\sum_{\gamma \in \Gamma_{e}(H)} \omega(\gamma)\left[e+c_{\gamma}\right]+\sum_{\gamma^{\prime} \in \Gamma(H) \backslash \Gamma_{e}(H)} \omega\left(\gamma^{\prime}\right)\left[\gamma^{\prime}\right]
\end{aligned}
$$

in $H_{1}\left(H ; \mathbb{Z}_{2}\right)$. This implies that if $\omega$ is not weakly balanced on $e$, then $\omega$ is not totally balanced on $\Gamma(H)$ over $\mathbb{Z}_{2}$.

Remark 3.3. A totally balanced weight $\omega$ on $\Gamma(H)$ over $\mathbb{Z}_{2}$ is not always weakly balanced on any pair of adjacent edges of $H$. For example, let $\omega$ be a weight on $\Theta_{3}$ (see Example 4.3) over $\mathbb{Z}_{2}$ defined by $\omega(\gamma)=1$ for any cycle $\gamma \in \Gamma\left(\Theta_{3}\right)$. It is easy to see that $\omega$ is totally balanced, but not weakly balanced, on each pair of adjacent edges of $\Theta_{3}$.

Proof of Theorem 2.3. (1) Let $f$ and $g$ be two spatial embeddings of $G$ which are edge-homotopic such that

$$
\omega_{1}(\gamma) \omega_{2}\left(\gamma^{\prime}\right) \operatorname{lk}\left(f(\gamma), f\left(\gamma^{\prime}\right)\right)=\omega_{1}(\gamma) \omega_{2}\left(\gamma^{\prime}\right) \operatorname{lk}\left(g(\gamma), g\left(\gamma^{\prime}\right)\right)=0
$$

in $\mathbb{Z}$ for any $\gamma \in \Gamma\left(G_{1}\right)$ and $\gamma^{\prime} \in \Gamma\left(G_{2}\right)$. First we show that if $f$ is transformed into $g$ by self crossing changes on $f\left(G_{1}\right)$ and ambient isotopies, then $\beta_{\omega_{1}, \omega_{2}}(f)=$ $\beta_{\omega_{1}, \omega_{2}}(g)$. In the same way as the proof of Theorem 2.2. we may consider three spatial embeddings $f, g$ and $h$ of $G$ and the knot $J_{h}$ as illustrated in Figure 3.2 . 
Then, by the same calculation in the proof of Theorem 2.2, we have that

$$
\begin{aligned}
\beta_{\omega_{1}, \omega_{2}}(f)-\beta_{\omega_{1}, \omega_{2}}(g) & \equiv-\left(\sum_{\gamma \in \Gamma_{e}\left(G_{1}\right)} \omega_{1}(\gamma)\right) \sum_{\gamma^{\prime} \in \Gamma\left(G_{2}\right)} \omega_{2}\left(\gamma^{\prime}\right) \mathrm{lk}\left(h\left(\gamma^{\prime}\right), J_{h}\right)^{2} \\
& \equiv\left(\sum_{\gamma \in \Gamma_{e}\left(G_{1}\right)} \omega_{1}(\gamma)\right) \sum_{\gamma^{\prime} \in \Gamma\left(G_{2}\right)} \omega_{2}\left(\gamma^{\prime}\right) \operatorname{lk}\left(h\left(\gamma^{\prime}\right), J_{h}\right) \\
& \equiv\left(\sum_{\gamma \in \Gamma_{e}\left(G_{1}\right)} \omega_{1}(\gamma)\right) \operatorname{lk}\left(\sum_{\gamma^{\prime} \in \Gamma\left(G_{2}\right)} \omega_{2}\left(\gamma^{\prime}\right) h\left(\gamma^{\prime}\right), J_{h}\right) .
\end{aligned}
$$

If $\omega_{1}$ is totally balanced on $\Gamma\left(G_{1}\right)$, then by Lemma 3.2 it is weakly balanced on any edge $e$ of $G_{1}$. This implies that $\beta_{\omega_{1}, \omega_{2}}(f)=\beta_{\omega_{1}, \omega_{2}}(g)$. If $\omega_{2}$ is totally balanced on $\Gamma\left(G_{1}\right)$, then we have that

$$
\operatorname{lk}\left(\sum_{\gamma^{\prime} \in \Gamma\left(G_{2}\right)} \omega_{2}\left(\gamma^{\prime}\right) h\left(\gamma^{\prime}\right), J_{h}\right) \equiv \mathrm{lk}\left(0, J_{h}\right)=0 .
$$

Therefore this also implies that $\beta_{\omega_{1}, \omega_{2}}(f)=\beta_{\omega_{1}, \omega_{2}}(g)$. In the same way we can show that if $f$ is transformed into $g$ by self crossing changes on $f\left(G_{2}\right)$ and ambient isotopies, then $\beta_{\omega_{1}, \omega_{2}}(f)=\beta_{\omega_{1}, \omega_{2}}(g)$. Thus we have that $\beta_{\omega_{1}, \omega_{2}}$ is an edge-homotopy invariant.

(2) By considering the triple of spatial embeddings as illustrated in Figure 3.3, we can prove (2) in a similar way as the proof of (1). We also omit the details.

Since the Conway polynomial of a split link is zero, our invariants take the value zero for any split (2-component) spatial graph. Therefore if the value of our invariant of a spatial graph is not zero, then it is non-splittable up to edge (resp. vertex)-homotopy.

\section{INTEGER-VALUED INVARIANTS}

Let $G$ be a planar graph. An embedding $p: G \rightarrow S^{2}$ is said to be cellular if the closure of each of the connected components of $S^{2}-p(G)$ is homeomorphic to the disk. Then we regard the set of the boundaries of all of the connected components of $S^{2}-p(G)$ as a subset of $\Gamma(G)$ and denote it by $\Gamma_{p}(G)$. We say that $G$ admits a checkerboard coloring on $S^{2}$ if there exists a cellular embedding $p: G \rightarrow S^{2}$ such that we can color all of the connected components of $S^{2}-p(G)$ by two colors (black and white) so that any of the two components which are adjacent by an edge have distinct colors; see Figure 4.1. We denote the subset of $\Gamma_{p}(G)$ which corresponds to the black (resp. white) colored components by $\Gamma_{p}^{b}(G)\left(\operatorname{resp} . \Gamma_{p}^{w}(G)\right)$.

Proposition 4.1. Let $G$ be a planar graph which is not homeomorphic to $S^{1}$ and admits a checkerboard coloring on $S^{2}$ with respect to a cellular embedding $p: G \rightarrow$ $S^{2}$. Let $\omega_{p}$ be a weight on $\Gamma(G)$ over $\mathbb{Z}$ defined by

$$
\omega_{p}(\gamma)= \begin{cases}1 & \left(\gamma \in \Gamma_{p}^{b}(G)\right) \\ -1 & \left(\gamma \in \Gamma_{p}^{w}(G)\right) \\ 0 & \left(\gamma \in \Gamma(G) \backslash \Gamma_{p}(G)\right) .\end{cases}
$$

Then $\omega_{p}$ is weakly balanced on any edge of $G$. 

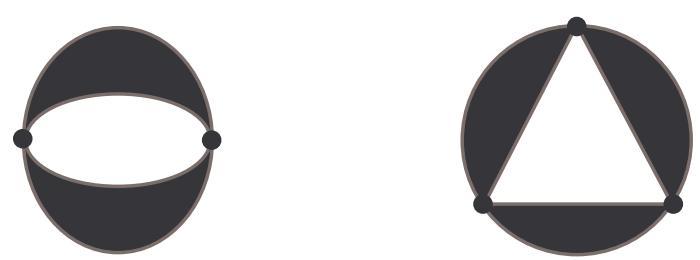

Figure 4.1

Proof. For any edge $e$ of $G$, there exist exactly two cycles $\gamma \in \Gamma_{p}^{b}(G)$ and $\gamma^{\prime} \in \Gamma_{p}^{w}(G)$ such that $e \subset \gamma$ and $e \subset \gamma^{\prime}$. Thus we have the result.

We call the weight $\omega_{p}$ in Proposition 4.1 a checkerboard weight. Thus by Proposition 4.1 and Theorem 2.2 (1), we can obtain an integer-valued edge-homotopy invariant as follows.

Theorem 4.2. Let $G=G_{1} \cup G_{2}$ be a disjoint union of two connected planar graphs such that $G_{i}$ is not homeomorphic to $S^{1}$ and admits a checkerboard coloring on $S^{2}$ with respect to a cellular embedding $p_{i}: G_{i} \rightarrow S^{2}(i=1,2)$. Let $\omega_{p_{i}}$ be a checkerboard weight on $\Gamma\left(G_{i}\right)$ over $\mathbb{Z}(i=1,2)$ and $f$ a spatial embedding of $G$ such that

$$
\omega_{p_{1}}(\gamma) \omega_{p_{2}}\left(\gamma^{\prime}\right) \operatorname{lk}\left(f(\gamma), f\left(\gamma^{\prime}\right)\right)=0
$$

in $\mathbb{Z}$ for any $\gamma \in \Gamma\left(G_{1}\right)$ and $\gamma^{\prime} \in \Gamma\left(G_{2}\right)$. Then $\beta_{\omega_{p_{1}}, \omega_{p_{2}}}(f)$ is an integer-valued edge-homotopy invariant of $f$.

Example 4.3. Let $\Theta_{n}$ be a graph with two vertices $u$ and $v$ and $n$ edges $e_{1}, e_{2}, \ldots$, $e_{n}$, each of which joins $u$ and $v$. A spatial embedding of $\Theta_{n}$ is called a (spatial) theta $n$-curve or simply a theta curve if $n=3$. For $n \geq 2$, we denote that a cycle of $\Theta_{n}$ consists of two edges $e_{i}$ and $e_{j}$ by $\gamma_{i j}(i<j)$. Then it is clear that $\Theta_{n}$ admits a cellular embedding $p: \Theta_{n} \rightarrow S^{2}$ so that

$$
\Gamma_{p}\left(\Theta_{n}\right)=\left\{\gamma_{12}, \gamma_{23}, \ldots, \gamma_{n-1, n}, \gamma_{1 n}\right\} .
$$

Moreover, for $m \geq 1, \Theta_{2 m}$ admits a checkerboard coloring on $S^{2}$ so that

$$
\begin{aligned}
\Gamma_{p}^{b}\left(\Theta_{2 m}\right) & =\left\{\gamma_{12}, \gamma_{34}, \ldots, \gamma_{2 m-1,2 m}\right\}, \\
\Gamma_{p}^{w}\left(\Theta_{2 m}\right) & =\left\{\gamma_{23}, \gamma_{45}, \ldots, \gamma_{2 m-2,2 m-1}, \gamma_{1,2 m}\right\} .
\end{aligned}
$$

Now let $G$ be a disjoint union of two copies of $\Theta_{4}$, each of which admits a checkerboard coloring on $S^{2}$ with respect to the cellular embedding $p$ as above. Let $\omega_{p}$ be a checkerboard weight on $\Gamma\left(\Theta_{4}\right)$ over $\mathbb{Z}$ and $g_{1}$ a spatial embedding of $G$ as illustrated in Figure 4.2. We can see that any of the 2-component constituent links of $g_{1}$ has a zero linking number. More precisely, $g_{1}$ contains exactly one non-trivial 2-component link $L=g_{1}\left(\gamma_{14}\right) \cup g_{1}\left(\gamma_{14}^{\prime}\right)$ whose linking number is zero. Thus by Theorem 4.2 we have that $\beta_{\omega_{p}, \omega_{p}}\left(g_{1}\right)$ is an integer-valued edge-homotopy invariant of $g_{1}$. Then, by a direct calculation we have that $a_{3}(L)=2$, namely $\beta_{\omega_{p}, \omega_{p}}\left(g_{1}\right)=2$. Note that a 2-component link is link-homotopically trivial if and only if its linking number is zero [8]. This implies that $g_{1}$ is non-splittable up to edge-homotopy despite the fact that any of the constituent links of $g_{1}$ is link-homotopically trivial. 


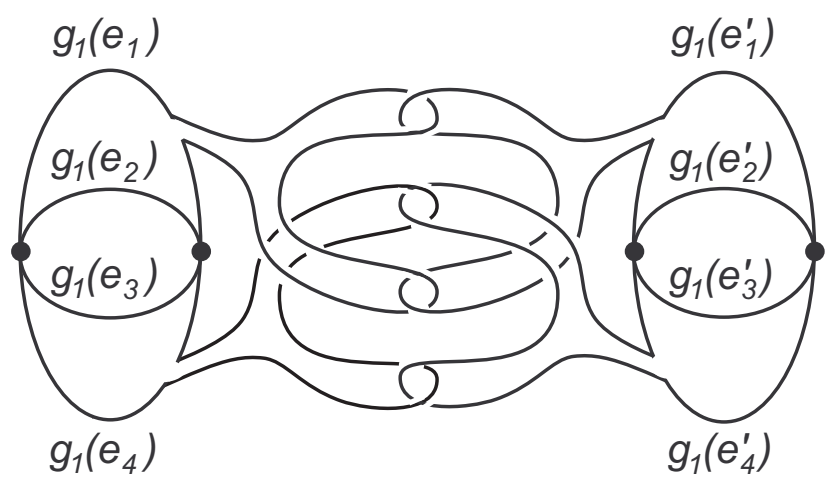

FiguRe 4.2

Moreover, for an integer $m$, let $g_{m}$ be a spatial embedding of $G$ as illustrated in Figure 4.3. If $m \neq 0$, we can see that $g_{m}$ contains exactly one non-trivial 2component link $L=g_{m}\left(\gamma_{14}\right) \cup g_{m}\left(\gamma_{14}^{\prime}\right)$ whose linking number is zero. Thus we also have that $\beta_{\omega_{p}, \omega_{p}}\left(g_{m}\right)$ is an integer-valued edge-homotopy invariant of $g_{m}$. Then, by a calculation we have that $a_{3}(L)=2 m$, namely $\beta_{\omega_{p}, \omega_{p}}\left(g_{m}\right)=2 m$. This implies that there exist infinitely many non-splittable spatial embeddings of $G$ up to edgehomotopy, all of whose constituent links are link-homotopically trivial.

Example 4.4. Let $H$ be a graph as illustrated in Figure 4.4, We denote the cycle of $H$ which contains $e_{i}$ and $e_{j}$ by $\gamma_{i j}(i<j)$. Let $G$ be a disjoint union of two copies of $H$ and $g_{1}$ a spatial embedding of $G$ as illustrated in Figure 4.5. This spatial embedding $g_{1}$ contains exactly one 4-component constituent link $L=$ $g_{1}\left(\gamma_{12} \cup \gamma_{34} \cup \gamma_{12}^{\prime} \cup \gamma_{34}^{\prime}\right)$. Note that if $g_{1}$ is split up to vertex-homotopy, then $L$ is split up to link-homotopy. Since $\left|\mu_{1234}(L)\right|=1$, where $\mu_{1234}$ denotes Milnor's $\mu$ invariant of length 4 of 4 -component links $[8$, we have that $L$ is non-splittable up to link-homotopy. Therefore we have that $g_{1}$ is non-splittable up to vertex-homotopy.

We can also prove this fact by our integer-valued vertex-homotopy invariant as follows. Let $\omega$ be a weight on $\Gamma(H)$ over $\mathbb{Z}$ defined by $\omega\left(\gamma_{14}\right)=\omega\left(\gamma_{23}\right)=1$, $\omega\left(\gamma_{13}\right)=\omega\left(\gamma_{24}\right)=-1$ and $\omega(\gamma)=0$ if $\gamma$ is a 2-cycle. Then it is easy to see that $\omega$ is weakly balanced on any pair of adjacent edges of $H$. We can see that $g_{1}$ contains exactly one non-trivial 2-component constituent link $M=g_{1}\left(\gamma_{14} \cup \gamma_{14}^{\prime}\right)$ with $\operatorname{lk}(M)=0$ and $a_{3}(M)=2$. Thus by Theorem 2.2 (2) we have that $\beta_{\omega, \omega}\left(g_{1}\right)$ is an integer-valued vertex-homotopy invariant of $g_{1}$ and $\beta_{\omega, \omega}\left(g_{1}\right)=2$. This implies that $g_{1}$ is non-splittable up to vertex-homotopy.

Moreover, let $g_{m}$ be a spatial embedding of $G$ as illustrated in Figure 4.5, which can be constructed in the same way as in Example 4.3. Then we can see that $\beta_{\omega, \omega}\left(g_{m}\right)$ is an integer-valued vertex-homotopy invariant of $g_{m}$ and $\beta_{\omega, \omega}\left(g_{m}\right)=2 m$. This implies that $g_{m}$ is non-splittable up to vertex-homotopy for any integer $m \neq 0$ and $g_{i}$ and $g_{j}$ are not vertex-homotopic for any $i \neq j$.

\section{Modulo two invariants}

Proposition 5.1. Let $G$ be a planar graph which is not homeomorphic to $S^{1}$ and $p: G \rightarrow S^{2}$ a cellular embedding. Let $\omega_{p}: \Gamma(G) \rightarrow \mathbb{Z}_{2}$ be a weight on $\Gamma(G)$ over $\mathbb{Z}_{2}$ 

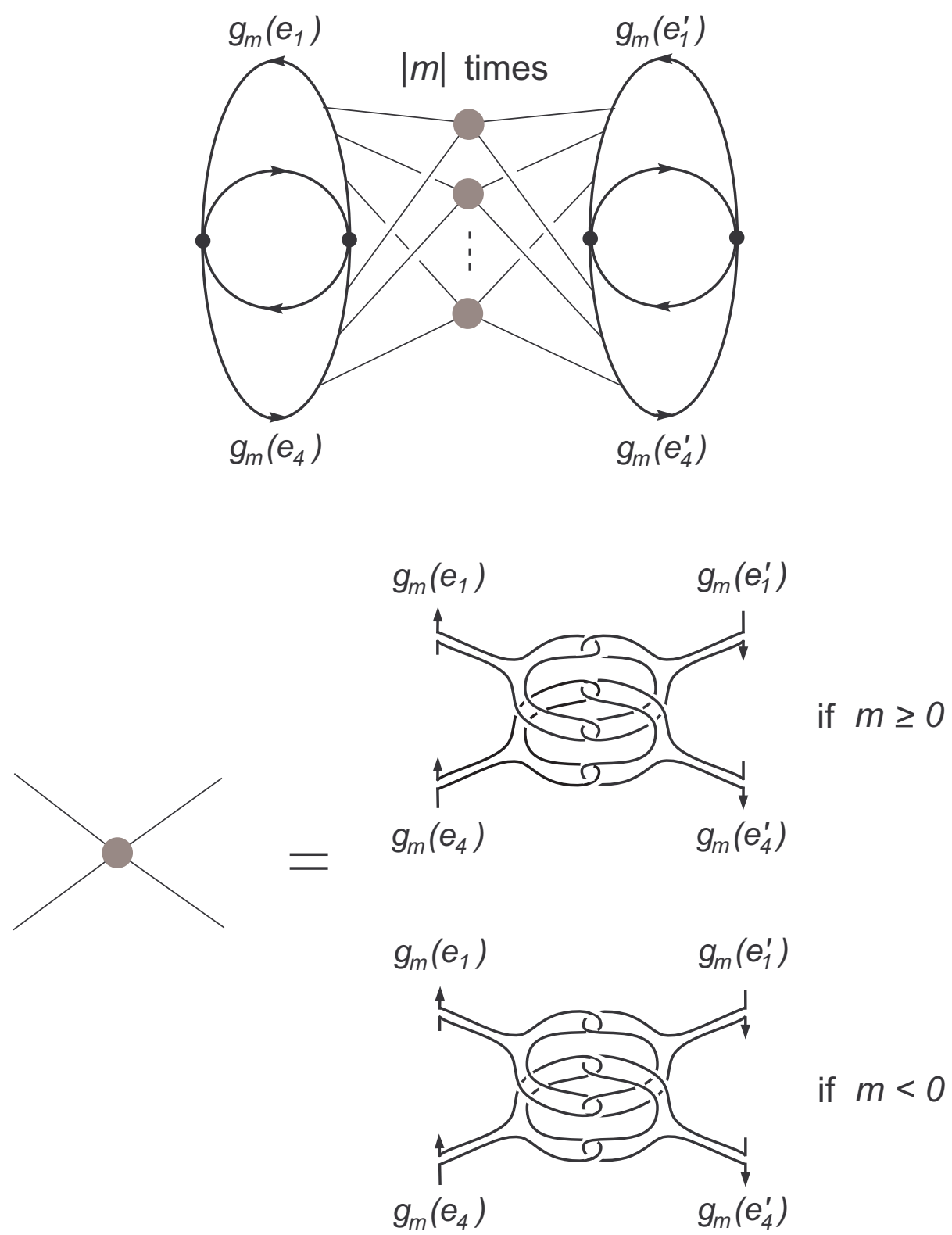

FiguRe 4.3

defined by

$$
\omega_{p}(\gamma)= \begin{cases}1 & \left(\gamma \in \Gamma_{p}(G)\right) \\ 0 & \left(\gamma \in \Gamma(G) \backslash \Gamma_{p}(G)\right) .\end{cases}
$$

Then $\omega_{p}$ is totally balanced. 


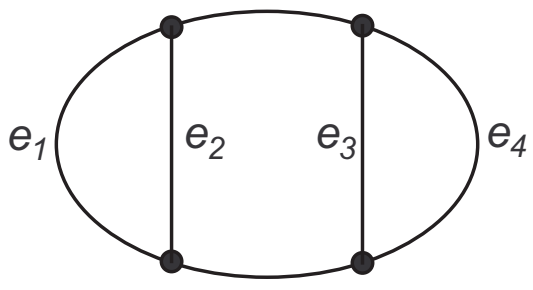

FigURE 4.4

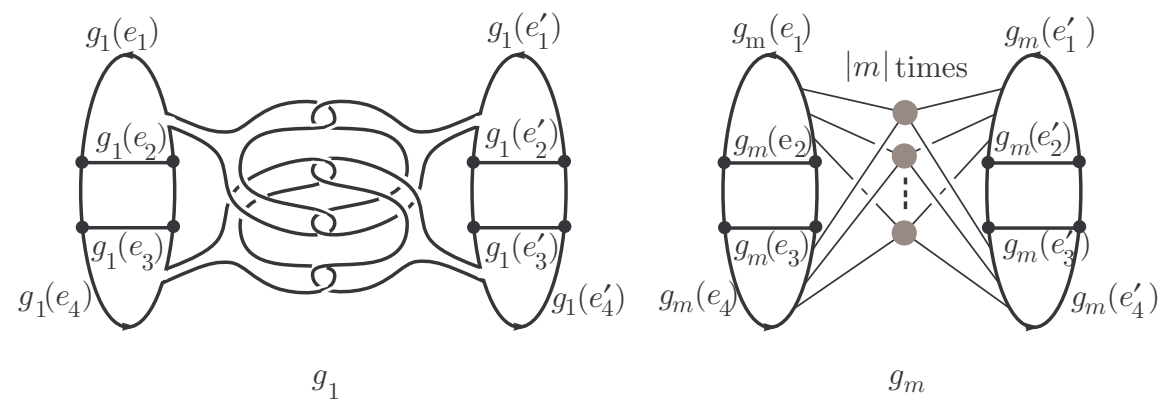

FigURE 4.5

Proof. It holds that

$$
\sum_{\gamma \in \Gamma(G)} \omega_{p}(\gamma)[\gamma]=\sum_{\gamma \in \Gamma_{p}(G)}[\gamma]=2\left[\sum_{e \in E(G)} e\right]=0
$$

in $H_{1}\left(G ; \mathbb{Z}_{2}\right)$, where $E(G)$ denotes the set of all edges of $G$. Thus we have the result.

Thus by Proposition 5.1 and Theorem 2.3 (1), we can obtain an edge-homotopy invariant as follows.

Theorem 5.2. Let $G=G_{1} \cup G_{2}$ be a disjoint union of two connected graphs such that $G_{1}$ is planar, not homeomorphic to $S^{1}$ and admits a cellular embedding $p_{1}: G_{1} \rightarrow S^{2}$. Let $\omega_{p_{1}}$ be a weight on $\Gamma\left(G_{1}\right)$ over $\mathbb{Z}_{2}$ as in Proposition 5.1, $\omega_{2}$ a weight on $\Gamma\left(G_{2}\right)$ over $\mathbb{Z}_{2}$ and $f$ a spatial embedding of $G$ such that

$$
\omega_{p_{1}}(\gamma) \omega_{2}\left(\gamma^{\prime}\right) \operatorname{lk}\left(f(\gamma), f\left(\gamma^{\prime}\right)\right)=0
$$

in $\mathbb{Z}$ for any $\gamma \in \Gamma\left(G_{1}\right)$ and $\gamma^{\prime} \in \Gamma\left(G_{2}\right)$. Then $\beta_{\omega_{p_{1}}, \omega_{2}}(f)$ is an edge-homotopy invariant of $f$.

Example 5.3. Let $G$ be a disjoint union of $\Theta_{3}$ and a circle $\gamma$. Let $\omega_{p}$ be a weight on $\Gamma\left(\Theta_{3}\right)$ over $\mathbb{Z}_{2}$ as in Proposition 5.1 with respect to a cellular embedding $p: \Theta_{3} \rightarrow S^{2}$ as in Example 4.3. and $\omega$ a weight on $\Gamma(\gamma)$ over $\mathbb{Z}_{2}$ defined by $\omega(\gamma)=1$. Let $g$ be a spatial embedding of $G$ as illustrated in Figure 5.1 (1). We can see that $g$ contains exactly one non-trivial 2-component link $L=g\left(\gamma_{13}\right) \cup g(\gamma)$ which is the Whitehead link, so $\operatorname{lk}(L)=0$ and $a_{3}(L)=1$. Thus by Theorem 5.2 we have that 
$\beta_{\omega_{p}, \omega}(g)$ is an edge-homotopy invariant of $g$ and $\beta_{\omega_{p}, \omega}(g)=1$. Namely $g$ is nonsplittable up to edge-homotopy despite the fact that any of the constituent links of $g$ is link-homotopically trivial.

Example 5.4. Let $G$ be a disjoint union of the complete bipartite graph on $3+3$ vertices $K_{3,3}$ and a circle $\gamma$. Let $\omega_{3,3}$ be a weight on $K_{3,3}$ over $\mathbb{Z}_{2}$ defined by $\omega_{3,3}\left(\gamma^{\prime}\right)=1$ if $\gamma^{\prime}$ is a 4 -cycle and 0 if $\gamma^{\prime}$ is a 6 -cycle. Let $\omega$ be a weight on $\Gamma(\gamma)$ over $\mathbb{Z}_{2}$ defined by $\omega(\gamma)=1$. Then it is not hard to see that $\omega_{3,3}$ is totally balanced and weakly balanced on any pair of adjacent edges of $K_{3,3}$. For a positive integer $m$, let $g_{m}$ be a spatial embedding of $G$ as illustrated in Figure 5.1 (2). Note that $g_{i}\left(K_{3,3}\right)$ and $g_{j}\left(K_{3,3}\right)$ are not vertex-homotopic for any $i \neq j\left[9\right.$; namely $g_{i}$ and $g_{j}$ are not vertex-homotopic for any $i \neq j$. Since all of the 2-component constituent links of $g_{m}$ are algebraically split, by Theorem 2.3 (2) we have that $\beta_{\omega_{3,3}, \omega}(g)$ is a vertexhomotopy invariant of $g_{m}$. Moreover we can see that there exists exactly one 4-cycle $\gamma^{\prime}$ of $K_{3,3}$ so that $L=g_{m}\left(\gamma \cup \gamma^{\prime}\right)$ is non-trivial. Since $L$ is the Whitehead link, we have that $\beta_{\omega_{3,3}, \omega}\left(g_{m}\right)=1$. Therefore $g_{m}$ is non-splittable up to vertex-homotopy despite the fact that any of the constituent links of $g$ is link-homotopically trivial.

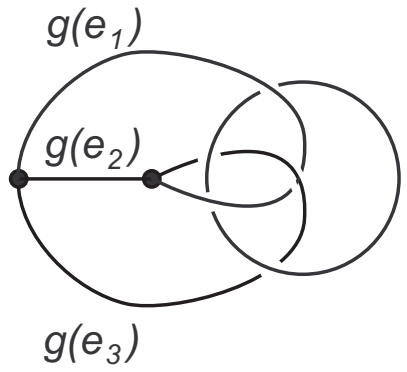

(1)

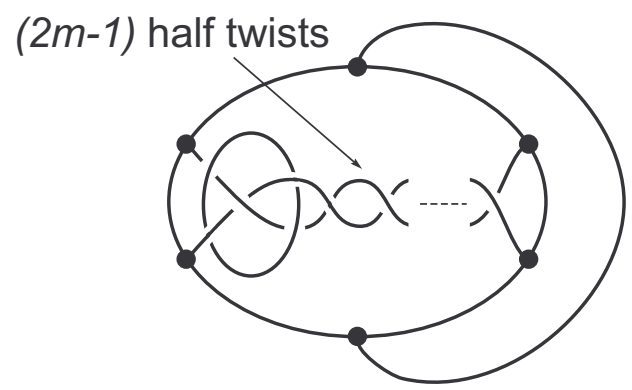

(2)

FigURE 5.1

Remark 5.5. The $\mathbb{Z}_{2}$-valued invariant in Theorem 2.3 cannot always be extended to an integer-valued one. For example,

(1) Let us consider the graph $G$ and the invariant $\beta_{\omega_{p}, \omega}$ as in Example 5.3, Let $f$ be a spatial embedding of $G$ as illustrated in Figure 5.2. We can see that $f$ is edge-homotopic to the trivial spatial embedding $h$ of $G$. But by a calculation we have that $\sum_{1 \leq i<j \leq 3} a_{3}\left(f\left(\gamma_{i j}\right), f(\gamma)\right)=-2$.

(2) Let $G$ be a disjoint union of $\Theta_{4}$ and a circle $\gamma$. Let $\omega_{p}$ be a checkerboard weight on $\Gamma\left(\Theta_{4}\right)$ over $\mathbb{Z}$ as in Example 4.3. Note that the modulo two reduction of a checkerboard weight is totally balanced. So by Theorem 2.3 (1), the modulo two reduction of $\sum_{\gamma_{i j} \in \Gamma\left(\Theta_{4}\right)} \omega_{p}\left(\gamma_{i j}\right) a_{3}\left(f\left(\gamma_{i j} \cup \gamma\right)\right)$ is an edge-homotopy invariant of a spatial embedding $f$ of $G$. Moreover, we can see that the integer-value $\sum_{\gamma_{i j} \in \Gamma\left(\Theta_{4}\right)} \omega_{p}\left(\gamma_{i j}\right) a_{3}\left(f\left(\gamma_{i j} \cup \gamma\right)\right)$ is invariant under the self crossing change on $f\left(\Theta_{4}\right)$ in the same way as in the proof of Theorem 2.2 (1). But this value may change under a self crossing change 


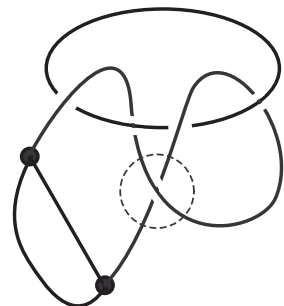

$f$

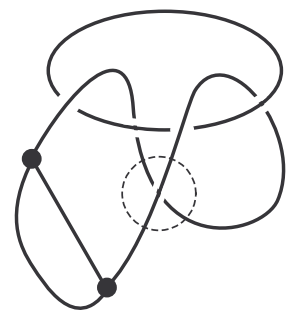

$h$

FiguRE 5.2

on $f(\gamma)$. For example, let $f$ and $g$ be two spatial embeddings of $G$ as illustrated in Figure 5.3 . We can see that $f$ is edge-homotopic to $g$. But by a calculation we have that

$$
\begin{aligned}
\sum_{\gamma_{i j} \in \Gamma\left(\Theta_{4}\right)} \omega_{p}\left(\gamma_{i j}\right) a_{3}\left(f\left(\gamma_{i j}\right), f(\gamma)\right) & =-1 \\
\sum_{\gamma_{i j} \in \Gamma\left(\Theta_{4}\right)} \omega_{p}\left(\gamma_{i j}\right) a_{3}\left(g\left(\gamma_{i j}\right), g(\gamma)\right) & =1 .
\end{aligned}
$$
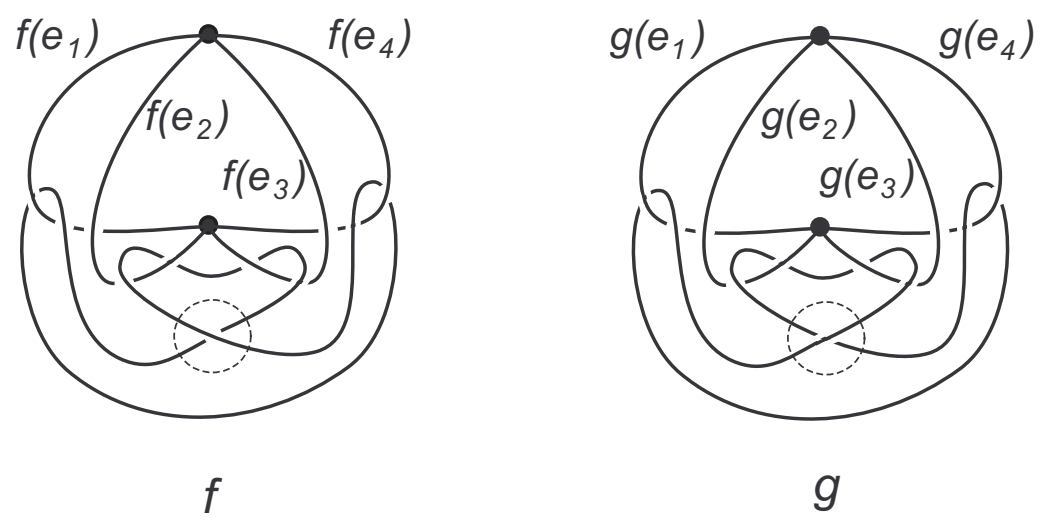

Figure 5.3

\section{Applying the Boundary of A Planar SURFACE}

Let $X$ be a disjoint union of a graph $G$ and a planar surface $F$ with boundary. Let $\omega$ be a weight on $\Gamma(G)$ over $\mathbb{Z}_{2}$ and $\varphi$ an embedding of $X$ into $S^{3}$ such that

$$
\omega(\gamma) \operatorname{lk}\left(\varphi(\gamma), \varphi\left(\gamma^{\prime}\right)\right)=0
$$

in $\mathbb{Z}$ for any $\gamma \in \Gamma(G)$ and $\gamma^{\prime} \in \Gamma(\partial F)$. Then we define $\beta_{\omega}(\varphi) \in \mathbb{Z}_{2}$ by

$$
\beta_{\omega}(\varphi) \equiv \sum_{\substack{\gamma \in \Gamma(G) \\ \gamma^{\prime} \in \Gamma(\partial F)}} \omega(\gamma) a_{3}\left(\varphi(\gamma), \varphi\left(\gamma^{\prime}\right)\right) \quad(\bmod 2) \text {. }
$$


Let $G$ be a disjoint union of a connected graph $G_{1}$ and a connected planar graph $G_{2}$. Let $f$ be a spatial embedding of $G$ and $p$ an embedding of $G_{2}$ into $S^{2}$. We denote the regular neighborhood of $p\left(G_{2}\right)$ in $S^{2}$ by $F\left(G_{2} ; p\right)$, which is a planar surface having $p\left(G_{2}\right)$ as a spine. Then the spatial embedding $f$ induces an embedding $\tilde{f}_{p}$ of the disjoint union $G_{1} \cup F\left(G_{2} ; p\right)$ into $S^{3}$, so that $\tilde{f}_{p}\left(G_{1}\right)=f\left(G_{1}\right)$ and $\tilde{f}_{p}\left(F\left(G_{2} ; p\right)\right)$ has $f\left(G_{2}\right)$ as a spine in the natural way. Note that such an induced embedding $\tilde{f}_{p}$ is not unique up to ambient isotopy. Let $\omega$ be a weight on $\Gamma\left(G_{1}\right)$ over $\mathbb{Z}_{2}$ so that

$$
\omega(\gamma) \operatorname{lk}\left(\tilde{f}_{p}(\gamma), \tilde{f}_{p}\left(\gamma^{\prime}\right)\right)=0
$$

in $\mathbb{Z}$ for any $\gamma \in \Gamma\left(G_{1}\right)$ and $\gamma^{\prime} \in \Gamma\left(\partial F\left(G_{2} ; p\right)\right)$. Then we have the following.

Theorem 6.1. If $f$ is split up to edge-homotopy, then $\beta_{\omega}\left(\tilde{f}_{p}\right)=0$ for any induced embedding $\tilde{f}_{p}$ of $G_{1} \cup F\left(G_{2} ; p\right)$.

Proof. By the assumption we have that $f$ is transformed into a split spatial embedding $u$ of $G$ by self crossing changes and ambient isotopies. Then each of the self crossing changes induces a self crossing change on $\tilde{f}_{p}\left(G_{1}\right)$ or a band-pass move [6] (see Figure 6.1) on $\tilde{f}_{p}\left(F\left(G_{2} ; p\right)\right)$. Namely $\tilde{f}_{p}$ can be transformed into an induced embedding $\tilde{u}_{p}$ of $G_{1} \cup F\left(G_{2} ; p\right)$ by such moves and ambient isotopies. Let $\tilde{g}_{p}$ be an embedding of $G_{1} \cup F\left(G_{2} ; p\right)$ into $S^{3}$ obtained from $\tilde{f}_{p}$ by a single self crossing change on $\tilde{f}_{p}\left(G_{1}\right)$ or a single band-pass move on $\tilde{f}_{p}\left(F\left(G_{2} ; p\right)\right)$. Then it still holds that

$$
\omega(\gamma) \operatorname{lk}\left(\tilde{g}_{p}(\gamma), \tilde{g}_{p}\left(\gamma^{\prime}\right)\right)=0
$$

in $\mathbb{Z}$ for any $\gamma \in \Gamma\left(G_{1}\right)$ and $\gamma^{\prime} \in \Gamma\left(\partial F\left(G_{2} ; p\right)\right)$.

Claim. $\beta_{\omega}\left(\tilde{f}_{p}\right)=\beta_{\omega}\left(\tilde{g}_{p}\right)$.

Assume that $\tilde{g}_{p}$ is obtained from $\tilde{f}_{p}$ by a single self crossing change on $\tilde{f}_{p}\left(G_{1}\right)$. Since it holds that

$$
\sum_{\gamma^{\prime} \in \Gamma\left(\partial F\left(G_{2} ; p\right)\right)}\left[\gamma^{\prime}\right]=0
$$

in $H_{1}\left(F\left(G_{2} ; p\right) ; \mathbb{Z}_{2}\right)$, we can see that $\beta_{\omega}\left(\tilde{f}_{p}\right)=\beta_{\omega}\left(\tilde{g}_{p}\right)$ in a similar way as the proof of Theorem 2.3 (1). Next we assume that $\tilde{g}_{p}$ is obtained from $\tilde{f}_{p}$ by a single bandpass move on $\tilde{f}_{p}\left(F\left(G_{2} ; p\right)\right)$. Then $\left.\tilde{g}_{p}\right|_{G_{1} \cup \partial F\left(G_{2} ; p\right)}$ is obtained from $\left.\tilde{f}_{p}\right|_{G_{1} \cup \partial F\left(G_{2} ; p\right)}$ by a single pass move [6] (see Figure 6.1) on $\tilde{f}_{p}\left(\partial F\left(G_{2} ; p\right)\right)$. We divide our situation into the following two cases.

Case 1. Four strings in the pass move belong to $\tilde{f}_{p}\left(\gamma_{1}^{\prime}\right)$ and $\tilde{f}_{p}\left(\gamma_{2}^{\prime}\right)$ for exactly two cycles $\gamma_{1}^{\prime}$ and $\gamma_{2}^{\prime}$ in $\Gamma\left(\partial F\left(G_{2} ; p\right)\right)$.

This pass move causes a single self crossing change on $\tilde{f}_{p}\left(\gamma_{1}^{\prime}\right)$ and a single self crossing change on $\tilde{f}_{p}\left(\gamma_{2}^{\prime}\right)$. Then the separated components that result from smoothing each of the self crossings are orientation-reversing parallel knots; see Figure 6.2. So the difference between $\beta_{\omega}\left(\tilde{f}_{p}\right)$ and $\beta_{\omega}\left(\tilde{g}_{p}\right)$ is cancelled out in a similar way as in the proof of Theorem 2.2 (1). Thus we have that $\beta_{\omega}\left(\tilde{f}_{p}\right)=\beta_{\omega}\left(\tilde{g}_{p}\right)$.

Case 2. Four strings in the pass move belong to $\tilde{f}_{p}\left(\gamma^{\prime}\right)$ for a cycle $\gamma^{\prime}$ in $\Gamma\left(\partial F\left(G_{2} ; p\right)\right)$.

It is known that a pass move on the same component of a proper link $L=$ $J_{1} \cup J_{2} \cup \cdots \cup J_{n}$ preserves $\overline{\operatorname{Arf}}(L) \equiv \operatorname{Arf}(L)-\sum_{i=1}^{n} \operatorname{Arf}\left(J_{i}\right) \in \mathbb{Z}_{2}$ (cf. [16])

\footnotetext{
${ }^{3}$ The value of $\overline{\operatorname{Arf}}(L)$ is called the reduced Arf invariant of $L$ [15].
} 
Especially, if $n=2$, then $a_{3}(L) \equiv \overline{\operatorname{Arf}}(L)(\bmod 2)$ [12, Lemma 3.5 (ii)]. Therefore in this case the pass move preserves $\omega(\gamma) a_{3}\left(\tilde{f}_{p}(\gamma), \tilde{f}_{p}\left(\gamma^{\prime}\right)\right)$ for any cycle $\gamma \in \Gamma\left(G_{1}\right)$. This implies that $\beta_{\omega}\left(\tilde{f}_{p}\right)=\beta_{\omega}\left(\tilde{g}_{p}\right)$.

Now by the argument above, we have that $\beta_{\omega}\left(\tilde{f}_{p}\right)=\beta_{\omega}\left(\tilde{u}_{p}\right)$. Then, each 2component link $\tilde{u}_{p}\left(\gamma \cup \gamma^{\prime}\right)$ is split for any $\gamma \in \Gamma\left(G_{1}\right)$ and $\gamma^{\prime} \in \Gamma\left(\partial F\left(G_{2} ; p\right)\right)$ because $u$ is split. Therefore we have that $\beta_{\omega}\left(\tilde{f}_{p}\right)=\beta_{\omega}\left(\tilde{u}_{p}\right)=0$. This completes the proof.
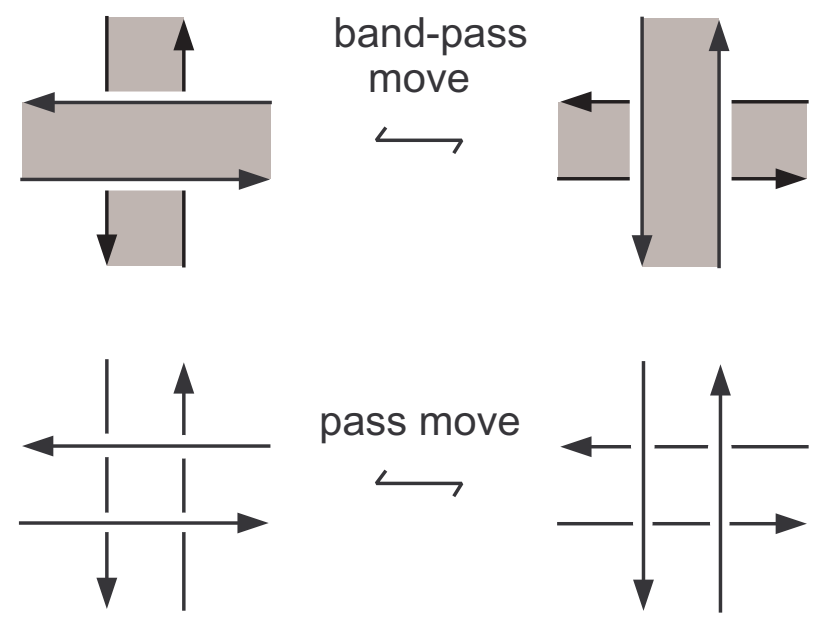

FiguRE 6.1

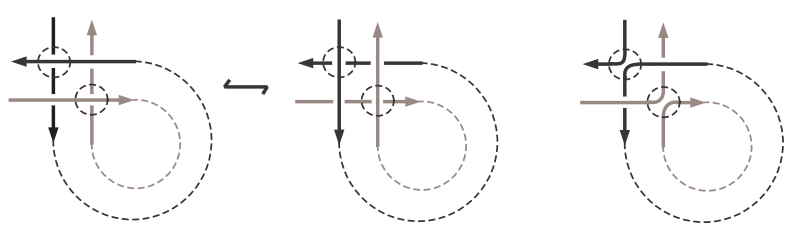

FiguRe 6.2

Example 6.2. Let $G$ be a disjoint union of a circle $\gamma$ and the handcuff graph (resp. 2-bouquet) $G_{2}$. Let $\omega$ be a weight on $\Gamma(\gamma)$ over $\mathbb{Z}_{2}$ defined by $\omega(\gamma)=1$. We fix an embedding $p: G_{2} \rightarrow S^{2}$ and take a regular neighborhood $F\left(G_{2} ; p\right)$ as illustrated in Figure 6.3 (1) (resp. (2)).

Let $f$ be a spatial embedding of $G$ as illustrated in Figure 2.1 (1) (resp. (2)). Let us take an induced embedding $\tilde{f}_{p}: \gamma \cup F\left(G_{2} ; p\right) \rightarrow S^{3}$ as illustrated in Figure 6.4 (1) (resp. (2)). Note that $\operatorname{lk}\left(\tilde{f}_{p}(\gamma), \tilde{f}_{p}\left(\gamma^{\prime}\right)\right)=0$ for any $\gamma^{\prime} \in \Gamma\left(\partial F\left(G_{2} ; p\right)\right)$. Then it can be calculated that $\beta_{\omega}\left(\tilde{f}_{p}\right)=1$. Thus by Theorem 6.1 we have that $f$ is non-splittable up to edge-homotopy.

\section{ACKNOWLEDGMENT}

The authors are very grateful to Professor Hitoshi Murakami for his hospitality at the Tokyo Institute of Technology where this work was conducted. 


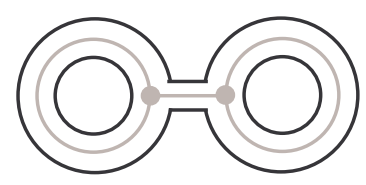

(1)

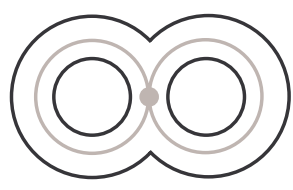

(2)

FiguRE 6.3

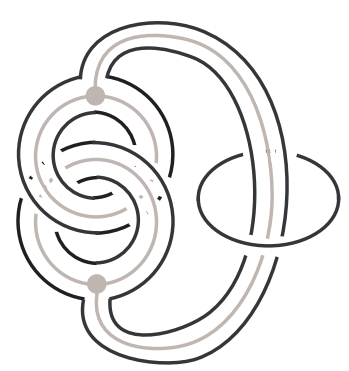

(1)

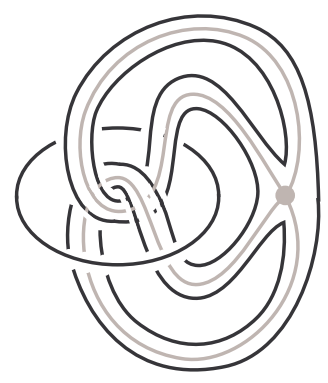

(2)

FiguRE 6.4

\section{REFERENCES}

[1] T. D. Cochran, Concordance invariance of coefficients of Conway's link polynomial, Invent. Math. 82 (1985), 527-541. MR87c:57002

[2] N. Habegger and X. -S. Lin, The classification of links up to link-homotopy, J. Amer. Math. Soc. 3 (1990), 389-419. MR91e:57015

[3] R. Hartley, The Conway potential function for links, Comment. Math. Helv. 58 (1983), 365378. MR $85 \mathrm{~h}: 57006$

[4] F. Hosokawa, On $\nabla$-polynomials of links, Osaka Math. J. 10 (1958), 273-282. MR21:1606

[5] J. Hoste, The first coefficient of the Conway polynomial, Proc. Amer. Math. Soc. 95 (1985), 299-302. MR:86m:57009

[6] L. H. Kauffman, Formal knot theory, Mathematical Notes, 30, Princeton University Press, Princeton, NJ, 1983. MR85b:57006

[7] J. P. Levine, An approach to homotopy classification of links, Trans. Amer. Math. Soc. 306 (1988), 361-387. MR88m:57008

[8] J. Milnor, Link groups, Ann. of Math. (2) 59 (1954), 177-195. MR:17:70e

[9] T. Motohashi and K. Taniyama, Delta unknotting operation and vertex homotopy of graphs in $R^{3}$, KNOTS '96 (Tokyo), 185-200, World Sci. Publishing, River Edge, NJ, 1997. MR99i:57021

[10] R. Nikkuni, Delta link-homotopy on spatial graphs, Rev. Mat. Complut. 15 (2002), 543-570. MR 2004d:57013

[11] R. Nikkuni, Edge-homotopy classification of spatial complete graphs on four vertices, J. Knot Theory Ramifications 13 (2004), 763-777. MR2005f:57008

[12] R. Nikkuni, Sharp edge-homotopy on spatial graphs, Rev. Mat. Complut. 18 (2005), 181-207. $\operatorname{MR} 2135538$ 
[13] Y. Ohyama and K. Taniyama, Vassiliev invariants of knots in a spatial graph, Pacific J. Math. 200 (2001), 191-205. MR2003a:57025

[14] N. Sato, Cobordisms of semiboundary links, Topology Appl. 18 (1984), 225-234. MR.86d:57010

[15] T. Shibuya, Self $\sharp$-unknotting operations of links, Mem. Osaka Inst. Tech. Ser. A 34 (1989), 9-17. MR92a:57014

[16] T. Shibuya and A. Yasuhara, Classification of links up to self pass-move, J. Math. Soc. Japan 55 (2003), 939-946. MR2004f:57016

[17] R. Sturm Beiss, The Arf and Sato link concordance invariants, Trans. Amer. Math. Soc. 322 (1990), 479-491. MR91m:57006

[18] K. Taniyama, Link homotopy invariants of graphs in $R^{3}$, Rev. Mat. Univ. Complut. Madrid 7 (1994), 129-144. MR 95f:57023

[19] K. Taniyama, Cobordism, homotopy and homology of graphs in $R^{3}$, Topology 33 (1994), 509-523. MR95h:57002

Department of Mathematics, University of California, San Diego, 9500 Gilman Drive, La Jolla, CALifornia 92093

E-mail address: tfleming@math.ucsd.edu

Institute of Human and Social Sciences, Faculty of Teacher Education, Kanazawa University, KaKuma-machi, KanaZaWa, IshikaWa, 920-1192, Japan

E-mail address: nick@ed.kanazawa-u.ac.jp 